\title{
Toxicity of the Hybrid Material Based on Low-Dimensional Structures of Aluminum Oxide
}

\author{
Olga V. Bakina ${ }^{1,2, a)}$, Elena A. Glazkova ${ }^{1,2, b)}$, Natalia V. Svarovskaya ${ }^{1,2,3, c),}$ \\ Aleksandr S. Lozhkomoev ${ }^{1,2, d)}$, Aleksandr M. Dygay ${ }^{4, e)}$, Aleksey A. Churin ${ }^{4, e)}$, \\ Marat I. Lerner ${ }^{1,2, \text { e) }}$, and Sergey G. Psakhie ${ }^{1,2, \text { f) }}$ \\ ${ }^{1}$ Institute of Strength Physics and Materials Science SB RAS, Tomsk, 634055, Russia \\ ${ }^{2}$ National Research Tomsk Polytechnic University, Tomsk, 634050, Russia \\ ${ }^{3}$ National Research Tomsk State University, Tomsk, 634050, Russia \\ ${ }^{4}$ Goldberg Research Institute of Pharmacology of the Tomsk Scientific Center SB RAMS, Tomsk, 634028, Russia

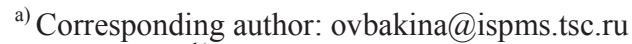 \\ b) eagl@ispms.tsc.ru \\ c)nvsv@ispms.tsc.ru \\ d) asl@ispms.tsc.ru \\ e) lerner@ispms.tsc.ru \\ f) sp@ms.tsc.ru
}

\begin{abstract}
Aluminum oxides of various morphology and phase composition, including pseudoboehmite, have wide application in medicine. The novel hybrid material from polymer fibers and low-dimensional structures of pseudoboehmite fixed on them can be used as a wound dressing due to its highly absorbent and adsorbent properties. The in vivo investigation of acute and chronic toxicity of the novel hybrid material after a single dermal application in the acute experiment for 8 and 24 hours with outbred male and female rats reveals no irritation or other pathological changes. The chronic toxicity testing of the material after multiple dermal applications at two doses causes no death of animals and no pathological changes in their general condition, weight dynamics, and visceral morphology. The macro- and microscopy of internal organs shows no pathological changes as compared to control animals. The results obtained bear witness to the low potential risk of toxicity when using the hybrid material as a wound dressing.
\end{abstract}

Keywords: hybrid material, low-dimensional structures of aluminum oxide, chronic toxicity, acute toxicity, local irritative effect

\section{INTRODUCTION}

Nano-sized and nanoporous aluminum oxide can potentially have many biomedical applications, including the development of new medication to treat skin wound in the form of a saline suspension and as a component of wound dressings [1,2]. As found in [3], aluminum oxide nanoparticles can penetrate the epithelial barrier and accumulate in target organs such as brain, spleen, kidney, heart, and others. Paper [4] notes that the aluminum oxide nanoparticles are more toxic to the brain than aluminum oxide particles of the micron size with the same surface chemical characteristics. At the same time the authors of [5] consider the toxicity of aluminum oxide to be low enough and dose-dependent.

Promising wound dressings are based on hybrid materials that consist of polymer fibers modified by lowdimensional structures of aluminum oxide. By adsorbing and inactivating microorganisms [6], this material helps to prevent bacterial colonization and re-infection of the wound and promotes active tissue regeneration. In this connection, there arises the question of evaluation of the safety of the hybrid material and its effect on human health. The present acute and chronic toxicity testing aims at determining the degree of damaging action of the hybrid

(C) 2014 AIP Publishing LLC 978-0-7354-1260-6/\$30.00 
material based on low-dimensional structures of aluminum oxide after single and multiple application on rats and at identifying most sensitive organs and body systems as well as the degree of damage reversibility.

\section{EXPERIMENTAL PROCEDURE}

The hybrid material was prepared as follows [7]. A sample of the polymer fibrous material from cellulose acetate was placed into an aqueous suspension $(1 \mathrm{wt} . \%)$ of nanoparticles of aluminum nitride composite Al/AlN, which were obtained by electrical explosion of aluminum in a nitrogen atmosphere. The reaction mixture is heated to $60^{\circ} \mathrm{C}$ for $20 \mathrm{~min}$ for water oxidation of $\mathrm{Al} / \mathrm{AlN}$ nanoparticles. Reaction products, namely, agglomerates of lowdimensional structures of aluminum oxide, were fixed to the surface of polymer fibers. The formed hybrid material was removed from the reaction medium and dried at $120^{\circ} \mathrm{C}$ for 4 hours.

The TEM images were obtained on finely powdered samples using a JEM-2100 transmission electron microscope (JEOL, Japan). The SEM analysis was performed with a LEO EVO 50 (Zeiss, Germany) electron microscope on samples covered with a thin silver layer. The X-ray diffraction analysis was performed on a Shimadzu XRD 6000 (Shimadzu, Japan) diffractometer at room temperature, $\mathrm{Cu} \mathrm{K} \alpha$ radiation was used.

The experiments were performed on adult outbred rats [8]. The animals were kept in accordance the rules of the European Convention for the Protection of Vertebrate Animals used for Experimental and Other Scientific Purposes, Strasbourg, 18.III.1986.

The acute toxicity tests were performed on 36 male and female rats weighting 180-200 $\mathrm{g}$ at the age of 22.5 months, which were divided into 4 experimental and 2 control groups. The hybrid material measuring $2 \times 2 \mathrm{~cm}$ was attached to the shaved back skin of rats. The exposure time was 8 and 24 hours.

The chronic toxicity tests were conducted on 60 male and female rats weighing 250-300 g, which were divided into 12 groups. The hybrid material measuring $1 \times 1 \mathrm{~cm}$ and $2 \times 2 \mathrm{~cm}$ was attached to the rat skin in the interscapular region during 30 days at the round-the-clock exposure. The recorded parameters were the general condition of animals, body temperature and weight, visceral morphology as well as local irritative effect. Reversibility of toxicity caused by the hybrid material was investigated on rats in 2 weeks after application.

The macro- and microscopy was performed on brain, heart, lung, liver, kidney, bladder, stomach, small and large intestine, pancreas, thyroid gland, spleen, thymus gland, adrenal gland, ovaries and uterus or testicles and prostate gland. The main parenchymatous organs after autopsy were isolated and weighed to determine weight indices. Tissue pieces were fixed in $10 \%$ formalin and embedded in paraffin. Deparaffinized sections were stained with hematoxylin and eosin. The local irritative effect of the hybrid material was histologically examined at the application site in rats.

\section{RESULTS AND DISCUSSION}

\section{Characteristics of the Hybrid Material}

The average particle size of the $\mathrm{Al} / \mathrm{AlN}$ precursor is $100 \mathrm{~nm}$ (Fig. 1(a)). The water reaction of $\mathrm{Al} / \mathrm{AlN}$ nanopowder results in low-dimensional structures of aluminum oxide in the form of folded nanosheets $5-10 \mathrm{~nm}$ in thickness and 150-300 $\mathrm{nm}$ in size, which are agglomerated as flower-like structures and bonded adhesively to polymer fibers [9]. (Figs. 1(b and c)). According to the X-ray analysis, they consist mainly of poorly crystallized pseudoboehmite AlOOH. The specific surface area is $320 \mathrm{~m}^{2} / \mathrm{g}$ and the average pore size 4-10 nm.

\section{Acute Toxicity Testing of the Hybrid Material in Rats}

After 8 and 24 hours of the hybrid material exposure rats have the skin of normal color without visible lesions and irritations. The animals are active, mobile, with normal motion coordination, usual frequency and depth of breathing, usual heart rhythm, normal fecal consistency, urinary frequency and color, with a good appetite and normal appearance. During the 14-day observation period, all animals gain in weight. The weight gain of the experimental animals is insignificantly different from that in the control groups. During the test, no mortality is recorded in either experimental or control group. 


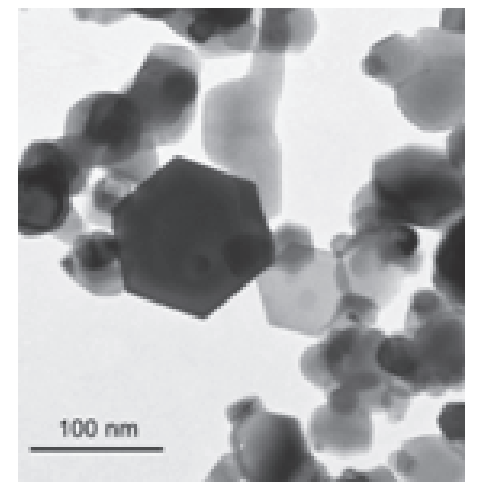

(a)

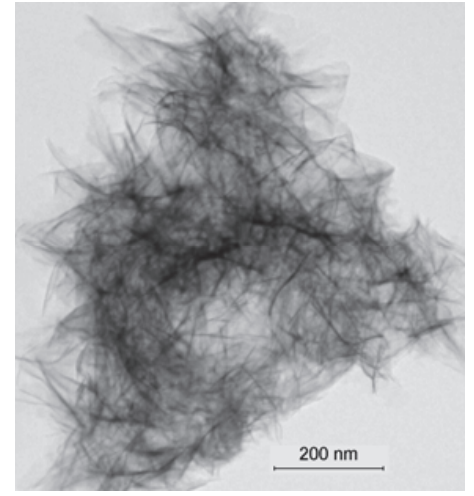

(b)

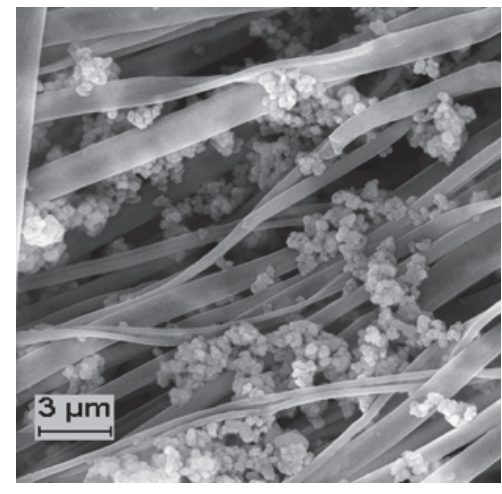

(c)

FIGURE 1. TEM image of Al/AIN particles (a), agglomerates of pseudoboehmite nanopetals (b), and SEM image of the hybrid material (c)

\section{Chronic Toxicity Testing of the Hybrid Material in Rats}

Weekly weighing shows a slower increment in body weight of male and female rats at the initial stage of the study. However, by the end of the experiment and in 2 weeks after the hybrid material application the weight gain of experimental and control males becomes the same.

After the 30-day course of the hybrid material application the dermal inspection reveals no skin irritation in rats. In 2 weeks after the application the hair of the control and experimental rats recover equally. The body temperature measurement of rats reveals no pathological changes. The recorded increase in the average body temperature in female rats after 1.5 months of the tested material application vanishes in the subsequent period of observation.

\section{Pathomorphological Study of Chronic Toxicity in Rats}

The macroscopic examination at autopsy on all rats shows no pathology of internal organs. In a month of the hybrid material application weight indices of all organs are indifferent from the control.

The microscopic examination of internal organs of almost all experimental rats shows that they have the usual structure and are indifferent from the control animals. Histological specimens of brain, hypophysis, heart, lung, liver, kidney, bladder, stomach, small intestine and large intestine, pancreas, thyroid gland, spleen, thymus gland, adrenal gland, uterus, ovaries, testicles, prostate gland reveal no differences from specimens of the control animals. The microscopic examination of the skin at the application site of the dressing material at two doses reveals no changes in the epidermis and dermis as compared to the control.

\section{CONCLUSION}

The investigation performed has revealed no acute and chronic toxicity of the hybrid material, no pathological changes in their general condition, weight dynamics and visceral morphology. The macro- and microscopy of internal organs has revealed no pathological changes as compared to the intact animals. The acute and chronic toxicity tests at the application site have shown that the hybrid material has no local irritative effect on the animal skin, which is indicative of the low potential risk of toxicity when using the hybrid material based on lowdimensional structures of aluminum oxide as an active component of wound dressings.

\section{ACKNOWLEDGEMENTS}

The present work was financially supported by Program of Fundamental Researches of the State Academies of Sciences for 2013-2020. 
The electron microscopy is carried out with the equipment of CUC NanoTech of the Institute of Strength Physics and Materials Sciences of the Siberian Branch of the Russian Academy of Sciences.

The acute and chronic toxicity tests of the hybrid material are carried out in the Goldberg Research Institute of Pharmacology of the Tomsk Scientific Center of the Siberian Branch of the Russian Academy of Medical Sciences (health certificate for laboratory animals No. 188-05 issued by the State Institution SCBT RAMS, Tomsk, Russia).

\section{REFERENCES}

1. G. E. Poinern, D. Fawcett, Y. J. Ng, N. Ali, R. K. Brundavanam, and Z. T. Jiang, J. Biomed. Nanotechnol. 6, 497-510 (2010).

2. M. Salerno, F. Caneva-Soumetz, L. Pastorino, N. Patra, A. Diaspro, and C. Ruggiero, IEEE Trans. Nanobioscie 12, 106-116 (2013).

3. A. Yokel and P. J. McNamara, Pharmacol Toxicol., 88, 159-167 (2001).

4. Q. Zhang, L. Xu, J. Wang, E. Sabbioni, L. Piao, M. Di Gioacchino, and Q. Niu, J. Biol. Regul. Homeost. Agents, 27, 365-375 (2013).

5. E. Dong, Y. Wang, S. T. Yang, Y. Yuan, H. Nie, Y. Chang, L. Wang, Y. Liu, and H. Wang, J. Nanosci. Nanotechnol. 11, 7848-7856 (2011).

6. M. I. Lerner, O. V. Bakina, E. A. Glazkova, A. S. Lozhkomoev, N. V. Svarovskaya, and S. G. Psakh'e, Inorg. Mat. 2, 488-492 (2011).

7. A. S. Lozhkomoev, E. A. Glazkova, E. G. Khorobraya, M. I. Lerner, A. N. Maltsev, and V. G. Podkovyrov, Russ. Phys. J. 56, 384-388 (2013).

8. A Guide for Experimental (Preclinical) Studies of New Pharmacological Substances, edited by R. U. Habriev (Meditsina, Moscow, 2005).

9. N. V. Svarovskaya, O. V. Bakina, E. A. Glazkova, M. I. Lerner, and S. G. Psakhie, Russ. J. Phys. Chem. A 84, 1566-1569 (2010). 